\title{
Classical symmetric functions in superspace
}

\author{
Patrick Desrosiers · Luc Lapointe · Pierre Mathieu
}

Received: 9 September 2005 / Accepted: 23 January 2006 / Published online: 11 July 2006

(C) Springer Science + Business Media, LLC 2006

\begin{abstract}
We present the basic elements of a generalization of symmetric function theory involving functions of commuting and anticommuting (Grassmannian) variables. These new functions, called symmetric functions in superspace, are invariant under the diagonal action of the symmetric group on the sets of commuting and anticommuting variables. In this work, we present the superspace extension of the classical bases, namely, the monomial symmetric functions, the elementary symmetric functions, the completely symmetric functions, and the power sums. Various basic results, such as the generating functions for the multiplicative bases, Cauchy formulas, involution operations as well as the combinatorial scalar product are also generalized.
\end{abstract}

Keywords Symmetric function $\cdot$ Superspace

\section{Introduction}

Superspace is an extension of Euclidean space in $N$ variables involving anticommuting variables. Its coordinates $\left(x_{1}, \ldots x_{N}, \theta_{1}, \ldots \theta_{N}\right)$ obey the relations $x_{i} x_{j}=$ $x_{j} x_{i}, x_{i} \theta_{j}=\theta_{j} x_{i}$, and $\theta_{i} \theta_{j}=-\theta_{j} \theta_{i}$. Functions in superspace, also called superfunctions, are thus functions of two types of variables. For instance, when $N=2$, all

P. Desrosiers

Department of Mathematics and Statistics, The University of Melbourne, Parkville, Australia, 3010

e-mail: P.Desrosiers@ms.unimelb.edu.au

\section{Lapointe}

Instituto de Matemática y Física, Universidad de Talca, Casilla 747, Talca, Chile

e-mail: lapointe@inst-mat.utalca.cl

P. Mathieu

Département de physique, de génie physique et d'optique, Université Laval, Québec, Canada, G1K 7P4

e-mail: pmathieu@phy.ulaval.ca 
functions are combinations of the following expressions

$$
f_{0}\left(x_{1}, x_{2}\right), \quad \theta_{1} f_{1}\left(x_{1}, x_{2}\right)+\theta_{2} f_{2}\left(x_{1}, x_{2}\right), \quad \theta_{1} \theta_{2} f_{3}\left(x_{1}, x_{2}\right)
$$

where the $f_{i}$ 's stand for arbitrary functions of $x_{1}$ and $x_{2}$. Functions of the second type are fermionic (alternatively said to be odd) while those of the first and third types are bosonic (even).

The aim of this work is to lay down the foundation of a symmetric function theory in superspace, where by a symmetric function in superspace, we understand a function invariant under the simultaneous interchange of $x_{i} \leftrightarrow x_{j}$ and $\theta_{i} \leftrightarrow \theta_{j}$ for any $i, j$. Examples when $N=2$ of symmetric polynomials in superspace are

$$
x_{1}^{2} x_{2}^{2}, \quad \theta_{1} x_{1}^{4}+\theta_{2} x_{2}^{4}, \quad \theta_{1} x_{2}^{2}+\theta_{2} x_{1}^{2}, \quad \theta_{1} \theta_{2}\left(x_{1}^{3} x_{2}-x_{1} x_{2}^{3}\right) .
$$

The enforced interconnection between the transformation properties of the bosonic and the fermionic variables is what makes the resulting objects most interesting and novel.

The first step in the elaboration of a theory of symmetric polynomials in superspace is the introduction of a proper labeling for bases of the ring of symmetric superpolynomials. This generalization of the concept of partitions, which was called superpartition in [4], turns out to be equivalent to what is known as a MacMahon standard diagram in [16]. With this concept in hand, the construction of the superextension of the symmetric monomial basis (supermonomial basis for short) is rather immediate [4]. From there on, the natural route for extending to superspace the multiplicative classical symmetric functions, such as elementary, homogeneous and power sum symmetric functions, is via the extension of their generating functions. The central point of this extension lies in the observation that the replacement $t x_{i} \rightarrow t x_{i}+\tau \theta_{i}$, where $t$ is the usual counting variable and $\tau$ is an anticommuting parameter, which lifts the generating functions directly to superspace, yields the "appropriate" bases. That is, the bases that are obtained have properties that extend those satisfied by their classical counterparts, such as for instance orthogonality relation and determinantal formulas. An even more convincing argument as to why this is the right symmetric function theory in superspace comes from its connection with an $N$-body problem in supersymmetric quantum mechanics involving a parameter $\beta$ (see e.g., [4, 8] and references therein). The eigenfunctions of the Hamiltonian of this model are superspace generalizations of Jack polynomials that specialize to various of the bases presented in this article, just as Jack polynomials specialize to classical bases of symmetric function theory [6]. Moreover, using a $\beta$-generalization of the results of this article, a purely combinatorial definition for these Jack polynomials in superspace can remarkably be obtained (see [10] for these developments).

The article is organized as follows. Section 2 first introduces the concept of superpartition. Then relevant results concerning the Grassmann algebra and symmetric superpolynomials are reviewed. A simple interpretation of the latter, in terms of differentials forms, is also given. This section also includes the definition of monomials in superspace and a formula for their products.

Our main results are presented in Section 3. It contains the superspace analog of the classical elementary symmetric functions, completely symmetric functions and Springer 
power-sum bases. The generating function for each of them is displayed. We point out at this stage an interesting connection between superpolynomials and de Rham complexes of symmetric $p$-forms. Determinantal expressions that generalize classical formulas relating the basis elements are presented. Furthermore, orthogonality and duality relations are also established.

As already indicated, this work concerns, to a large extent, a generalization of symmetric function theory. In laying down its foundation, we generalize a vast number of basic results from this theory which can be found for instance in [13] and [17] (Chapter 7). Clearly, the core of most of our derivations is bound to be a variation around the proofs of these older results. We have chosen not to refer everywhere to the relevant "zero-fermionic degree" version of the stated results to avoid overquoting. But we acknowledge our debt in that regard to these two classic references.

\section{Foundations}

\subsection{Superpartitions}

We recall that a partition $\lambda=\left(\lambda_{1}, \lambda_{2}, \ldots, \lambda_{\ell}\right)$ of $n$, also written as $\lambda \vdash n$, is an ordered set of integers such that: $\lambda_{1} \geq \lambda_{2} \geq \cdots \geq \lambda_{\ell} \geq 0$ and $\sum_{i=1}^{\ell} \lambda_{i}=n$. A particular juxtaposition of two partitions gives a superpartition.

Definition 1 ([4]). A superpartition $\Lambda$ in the $m$-fermion sector is a sequence of nonnegative integers separated by a semicolon such that the sequence before the semicolon is a partition with $m$ distinct parts, and such that the remaining sequence is a usual partition. That is,

$$
\Lambda=\left(\Lambda_{1}, \ldots, \Lambda_{m} ; \Lambda_{m+1}, \ldots, \Lambda_{N}\right)
$$

where $\quad \Lambda_{i}>\Lambda_{i+1} \geq 0 \quad$ for $\quad i=1, \ldots m-1 \quad$ and $\quad \Lambda_{j} \geq \Lambda_{j+1} \geq 0$ for $j=m+1, \ldots, N-1$.

Given $\Lambda=\left(\Lambda^{a} ; \Lambda^{s}\right)$, the partitions $\Lambda^{a}$ and $\Lambda^{s}$ are respectively called the antisymmetric and the symmetric components of $\Lambda$. (From now on, superscripts $a$ and $s$ refer respectively to strictly and weakly decreasing sequences of non-negative integers.) The bosonic degree (or simply degree) of $\Lambda$ is $|\Lambda|=\sum_{i=1}^{N} \Lambda_{i}$, while its fermionic degree (or sector) is $\bar{\Lambda}=m$. Note that, in the zero-fermion sector, the semicolon is usually omitted and $\Lambda$ reduces then to $\Lambda^{s}$.

We say that the ordered set $\Lambda$ in (2.1) is a superpartition of $(n \mid m)$ (a superparition of degree $n$ in the fermionic sector $m)$ if $|\Lambda|=n$ and $\underline{\Lambda}=m$, and write $\Lambda \vdash(n \mid m)$. The set composed of all superpartitions of $(n \mid m)$ is denoted $\operatorname{SPar}(n \mid m)$. When the fermionic degree is zero, we recover standard partitions: $\operatorname{SPar}(n \mid 0)=\operatorname{Par}(n)$.

We also define

$$
\operatorname{SPar}(n):=\bigcup_{m \geq 0} \operatorname{SPar}(n \mid m) \quad \text { and } \quad \operatorname{SPar}:=\bigcup_{m, n \geq 0} \operatorname{SPar}(n \mid m),
$$


with $\operatorname{SPar}(0 \mid 0)=\emptyset$ and $\operatorname{SPar}(0 \mid 1)=\{(0 ; 0)\}$. For example, we have

$$
\operatorname{SPar}(3 \mid 2)=\{(3,0 ; 0),(2,1 ; 0),(2,0 ; 1)(1,0 ; 2),(1,0 ; 1,1)\}
$$

Notice that $\operatorname{SPar}(n \mid m)$ is empty for all $n<m(m-1) / 2$.

The length of a superpartition is

$$
\ell(\Lambda):=\underline{\bar{\Lambda}}+\ell\left(\Lambda^{s}\right) \quad \text { with } \quad \ell\left(\Lambda^{s}\right):=\operatorname{Card}\left\{\Lambda_{i} \in \Lambda^{s}: \Lambda_{i}>0\right\} .
$$

With this definition, $\ell((1,0 ; 1,1))=2+2=4$ (i.e., a zero-entry in $\Lambda^{a}$ contributes to the length of $\Lambda$ ). To every superpartition $\Lambda$, we can also associate a unique partition $\Lambda^{*}$ obtained by deleting the semicolon and reordering its parts in non-increasing order. For instance,

$(5,2,1,0 ; 6,5,5,2,2,1)^{*}=(6,5,5,5,2,2,2,1,1,0)=(6,5,5,5,2,2,2,1,1)$.

From this, we can introduce another notation for superpartitions. A superpartition $\Lambda=\left(\Lambda^{a} ; \Lambda^{s}\right)$ can be viewed as the partition $\Lambda^{*}$ in which every part of $\Lambda^{a}$ is circled. If an entry $b$ of $\Lambda^{a}$ also occurs in $\Lambda^{s}$, then we circle the leftmost $b$ appearing in $\Lambda^{*}$. We shall use $C[\Lambda]$ when refering to such a circled partition. For instance,

$$
\Lambda=(3,1,0 ; 4,3,2,1) \Longleftrightarrow C[\Lambda]=(4,3,3,2,1,1,0)
$$

The notation $C[\Lambda]$ allows us to introduce a diagrammatic representation of superpartitions. To each $\Lambda$, we associate a diagram, denoted by $D[\Lambda]$. It is obtained by first drawing the Ferrers diagram associated to $C[\Lambda]$, that is, by drawing a diagram with $C[\Lambda]_{1}$ boxes in the first row, $C[\Lambda]_{2}$ boxes in the second row and so forth, all rows being left justified. In addition, if the $j$-th entry of $C[\Lambda]$ is circled, then we add a circle at the end of the $j$-th row of the diagram. We shall further denote by $\operatorname{sh}(D[\Lambda])$ the shape of $D[\Lambda]$ (including the circles). For example, with $\Lambda=(3,1,0 ; 4,3,2,1)$, we have as mentionned $C[\Lambda]=(4$, (3), 3, 2, (1), 1, (0)), and thus

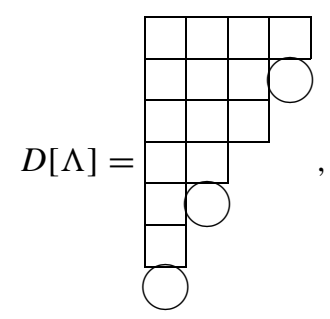

giving that $\operatorname{sh}(D[\Lambda])=(4,4,3,2,2,1,1)$.

The conjugate of a superpartition $\Lambda$, denoted by $\Lambda^{\prime}$, is the superpartition whose diagram is the transposed (with respect to the main diagonal) of that of $D[\Lambda]$. Hence, 祭 Springer 
$(3,1,0 ; 4,3,2,1)^{\prime}=(6,4,1 ; 3)$ since the transposed of the previous diagram is

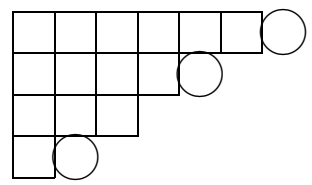

Obviously, the conjugation of any superpartition $\Lambda$ satisfies

$$
\left(\Lambda^{\prime}\right)^{\prime}=\Lambda \quad \text { and } \quad\left(\Lambda^{*}\right)^{\prime}=\left(\Lambda^{\prime}\right)^{*}
$$

Remark 2. The description of superpartitions using Ferrers diagram with some rows ending with a circle makes clear that superpartitions are equivalent to standard MacMahon diagrams, which are Ferrers diagrams with some corner cells marked (see for instance [16], Section 2.1.3). We shall nevertheless keep refering to superpartition as superpartitions to be consistent with our previous articles.

Furthermore, the notation $C[\Lambda]$ for a superpartition gives immediately that the overpartitions introduced recently in [3] are special cases of superpartitions. Indeed, overpartitions are circled superpartitions (with the circle replaced by an overbar) that do not contain a possible circled zero. If we denote by $s_{N}(n \mid m)$ the number of superpartitions of $(n \mid m)$ such that $\ell(\Lambda) \leq N$, then this connection implies that their generating function is

$$
\sum_{n, m, p \geq 0} s_{m+p}(n \mid m) z^{m} y^{p} q^{n}=\frac{(-z ; q)_{\infty}}{(y q ; q)_{\infty}} \quad \text { with } \quad(a ; q)_{\infty}:=\prod_{n \geq 0}\left(1-a q^{n}\right)
$$

To complete this subsection, we consider the natural ordering on superpartitions. We will first define it in terms of the Bruhat order on compositions, and then later, in Corollary 7, give a simpler characterization. Recall that a composition of $n$ is simply a sequence of non-negative integers whose sum is equal to $n$; in symbols $\mu=\left(\mu_{1}, \mu_{2}, \ldots\right) \in \operatorname{Comp}(n)$ iff $\sum_{i} \mu_{i}=n$ and $\mu_{i} \geq 0$ for all $i$. The Bruhat ordering on compositions is defined as follows. Given a composition $\lambda$, we let $\lambda^{+}$denote the partition obtained by reordering its parts in non-increasing order. Now, $\lambda$ can be obtained from $\lambda^{+}$by a sequence of permutations. Among all permutations $w$ such that $\lambda=w \lambda^{+}$, there exists a unique one, denoted $w_{\lambda}$, of minimal length. For two compositions $\lambda$ and $\mu$, we say that $\lambda \geq \mu$ if either $\lambda^{+}>\mu^{+}$in the usual dominance ordering or $\lambda^{+}=\mu^{+}$and $w_{\lambda} \leq w_{\mu}$ in the sense that the word $w_{\lambda}$ is a subword of $w_{\mu}$ (this is the Bruhat ordering on permutations of the symmetric group). Recall that for two partitions $\lambda$ and $\mu$ of the same degree, the dominance ordering is: $\lambda \geq \mu$ iff $\lambda_{1}+\cdots+\lambda_{k} \geq \mu_{1}+\cdots+\mu_{k}$ for all $k$.

Let $\Lambda$ be a superpartition of $(n \mid m)$. Then, to $\Lambda$ is associated a unique composition of $n$, denoted by $\Lambda^{c}$, obtained by replacing the semicolon in $\Lambda$ by a comma. We thus have $\operatorname{Spar}(n) \subset \operatorname{Comp}(n)$, which leads to a natural Bruhat ordering on superpartitions.

Definition 3 ([6]). Let $\Lambda, \Omega \in$ SPar. The Bruhat order, denoted by $\leq$, is defined such that $\Omega \leq \Lambda$ iff $\Omega^{c} \leq \Lambda^{c}$. 
For later purposes, we shall divide the Bruhat order into two orders, depending on whether or not the superpartitions reorder to the same partitions.

Definition 4. Let $\Lambda, \Omega \in$ SPar. The $S$ and $T$ orders are respectively defined as follows:

$$
\begin{aligned}
& \Omega \leq_{S} \Lambda \quad \text { if either } \Omega=\Lambda \text { or } \Omega^{*}<\Lambda^{*}, \\
& \Omega \leq_{T} \Lambda \text { if either } \Omega=\Lambda \text { or } \Omega^{*}=\Lambda^{*} \text { and } \Omega^{c}<\Lambda^{c} \text {. }
\end{aligned}
$$

In order to describe other characterizations of these orders, we need the following operators on compositions (or superpartitions):

$$
\begin{aligned}
& S_{i j}\left(\ldots, \lambda_{i}, \ldots, \lambda_{j}, \ldots\right)= \begin{cases}\left(\ldots, \lambda_{i}-1, \ldots, \lambda_{j}+1 \ldots\right) & \text { if } \lambda_{i}-\lambda_{j}>1 \\
\left(\ldots, \lambda_{i}, \ldots, \lambda_{j}, \ldots\right) & \text { otherwise },\end{cases} \\
& T_{i j}\left(\ldots, \lambda_{i}, \ldots, \lambda_{j}, \ldots\right)= \begin{cases}\left(\ldots, \lambda_{j}, \ldots, \lambda_{i}, \ldots\right) & \text { if } \lambda_{i}-\lambda_{j}>0 \\
\left(\ldots, \lambda_{i}, \ldots, \lambda_{j}, \ldots\right) & \text { otherwise }\end{cases}
\end{aligned}
$$

Remark 5. The $S$ order is precisely the ordering introduced in [4]. It differs from the more precise ordering of [5], which was called $\leq^{s}$. In [6], it is called the $h$ ordering. See also Appendix B of [7].

The next lemma will lead to a simpler characterization of the order on superpartitions.

Lemma $6([13,14])$. Let $\lambda$ and $\omega$ be two compositions of $n$. Then, $\lambda^{+}>\omega^{+}$iff there exists a sequence $\left\{S_{i_{1}, j_{1}}, \ldots, S_{i_{k}, j_{k}}\right\}$ such that

$$
\omega^{+}=S_{i_{1}, j_{1}} \ldots S_{i_{k}, j_{k}} \lambda^{+} .
$$

Similarly, $\lambda^{+}=\omega^{+}$and $\lambda>\omega$ iff there exists a sequence $\left\{T_{i_{1}, j_{1}}, \ldots, T_{i_{k}, j_{k}}\right\}$ such that

$$
\omega=T_{i_{1}, j_{1}} \ldots T_{i_{k}, j_{k}} \lambda
$$

Since a $T_{i, j}$ operation on a superpartition $\Lambda$ amounts to removing the circle from row $i$ in $D[\Lambda]$ and adding it to row $j$, the second part of this lemma can be translated for superpartitions as: $\Lambda>_{T} \Omega$ iff $\operatorname{sh}(D[\Lambda])>\operatorname{sh}(D[\Omega])$ in the dominance order, where we recall that $\operatorname{sh}(D[\Lambda])$ is the shape of $D[\Lambda]$ with the circles included. This provides a simpler way to understand the order on superpartitions.

Corollary 7. Let $\Lambda, \Omega \in$ SPar. We have that $\Omega \leq \Lambda$ iff $\Omega^{*}<\Lambda^{*}$ or $\Omega^{*}=\Lambda^{*}$ and $\operatorname{sh}(D[\Omega]) \leq \operatorname{sh}(D[\Lambda])$. 
At this stage, we are in a position to establish the fundamental property relating conjugation and Bruhat order which is that the Bruhat order is anti-conjugate (in the sense of the following proposition).

Proposition 8. Let $\Lambda, \Omega \in \operatorname{SPar}(n \mid m)$. Then

$$
\Lambda \geq \Omega \Longleftrightarrow \Omega^{\prime} \geq \Lambda^{\prime} \text {. }
$$

Proof: It suffices to prove the result for the $S$ and $T$ orderings. The case $\Lambda>_{S} \Omega$, that is, $\Lambda^{*}>\Omega^{*}$, is a well-known result on partitions (see for instance (1.11) of [13]). From Corollary 7 , the case $\Lambda>_{T} \Omega$ follows from the same argument.

Remark 9. Notice that we had before introduced as an alternative ordering the dominance ordering on superpartitions, denoted by $\leq_{D}$ and defined as follows: $\Omega \leq_{D} \Lambda$ if either $\Omega^{*}<\Lambda^{*}$ or $\Omega^{*}=\Lambda^{*}$ and $\Omega_{1}+\cdots+\Omega_{k} \leq \Lambda_{1}+\cdots \Lambda_{k}, \forall k$. The usefulness of this ordering in special contexts lies in its simple description in terms of inequalities. However, it is not the proper generalization of the dominance order on partitions. In fact, it is not as strict as the Bruhat ordering (i.e., more superpartitions are comparable in this order than in the Bruhat ordering). This follows from the second property of Lemma 6 which obviously implies that for superpartitions, the Bruhat ordering is a weak subposet of the dominance ordering, that is, $\Lambda \geq \Omega \Rightarrow \Lambda \geq{ }_{D} \Omega$. However the converse is not true. For instance, if $\Lambda=(5,2,1 ; 4,3,3)$ and $\Omega=(4,3,0 ; 5,3,2,1)$ we easily verify that $\Lambda>_{D} \Omega$. But since $\Lambda^{*}=\Omega^{*}, \operatorname{sh}(D[\Lambda])=(6,4,3,3,3,2)$ and $\operatorname{sh}(D[\Omega])=(5,5,4,3,2,1,1)$ we have that $\Lambda \ngtr \Omega$ by Corollary 7 . This corrects a loose implicit statement in [6] concerning the expected equivalence of these two orderings.

\subsection{Ring of symmetric polynomials in superspace}

Let $\mathscr{B}=\left\{B_{j}\right\}$ and $\mathscr{F}=\left\{F_{j}\right\}$ be the formal and infinite sets composed of all bosonic (commutative) and fermionic (anticommutative) quantities respectively. Thus, $\mathscr{O}=$ $\mathscr{B} \oplus \mathscr{F}$ is $\mathbb{Z}_{2}$-graded over any ring $\mathbb{A}$ when we identify ${ }^{0} \mathscr{O}$ with $\mathscr{B}$ and ${ }^{1} \mathscr{O}$ with $\mathscr{F}$ . $\mathscr{S}$ possesses a linear endomorphism $\hat{\Pi}$, called the parity operator, defined by

$$
\hat{\Pi}(s)=(-1)^{\hat{\pi}(s)}, \quad \text { where } \hat{\pi}(s)= \begin{cases}0, & s \in \mathscr{B}, \\ 1, & s \in \mathscr{F} .\end{cases}
$$

In other words, the product of two bosons gives a boson, the product of a boson and a fermion gives a fermion, and the product of two fermions gives a boson.

An example of such a structure is the Grassmann algebra over a unital ring $\mathbb{A}$, denoted $\mathscr{Y}_{M}(\mathbb{A})$. It is the algebra with identity $1 \in \mathbb{A}$ generated by the $M$ anticommuting elements $\theta_{1}, \ldots, \theta_{M}$. We shall need the following linear involution on the Grassmann algebra defined by:

$$
\overleftarrow{\theta_{j_{1}} \ldots \theta_{j_{m}}}:=\overrightarrow{\theta_{j_{m}} \ldots \theta_{j_{1}}} \quad \text { where } \quad \overrightarrow{\theta_{j_{1}} \ldots \theta_{j_{m}}}:=\theta_{j_{1}} \cdots \theta_{j_{m}}
$$


In words, the operator $\longleftarrow$ reverses the order of the anticommutative variables while is simply the identity map. (The explicit use of $\longrightarrow$ is not essential, but it will make many formulas more symmetric and transparent.) Using induction, we get

$$
\overleftarrow{\theta_{j_{1}} \ldots \theta_{j_{m}}}=(-1)^{m(m-1) / 2} \overrightarrow{\theta_{j_{1}} \ldots \theta_{j_{m}}}
$$

This result immediately implies the following simple properties.

Lemma 10. Let $\left\{\theta_{1}, \ldots, \theta_{N}\right\}$ and $\left\{\phi_{1}, \ldots, \phi_{N}\right\}$ be two sets of Grassmannian variables. Then

$$
\left(\theta_{j_{1}} \phi_{j_{1}}\right) \ldots\left(\theta_{j_{m}} \phi_{j_{m}}\right)=\overleftarrow{\left(\theta_{j_{1}} \ldots \theta_{j_{m}}\right)} \overrightarrow{\left(\phi_{j_{1}} \ldots \phi_{j_{m}}\right)}=\overleftrightarrow{\left(\theta_{j_{1}} \ldots \theta_{j_{m}}\right)} \overleftarrow{\left(\phi_{j_{1}} \ldots \phi_{j_{m}}\right)}
$$

and

$$
\overleftarrow{\left(\theta_{j_{1}} \ldots \theta_{j_{m}}\right)} \overline{\left(\phi_{j_{1}} \ldots \phi_{j_{m}}\right)}=\overline{\left(\phi_{j_{1}} \ldots \phi_{j_{m}}\right)} \overleftarrow{\left(\theta_{j_{1}} \ldots \theta_{j_{m}}\right)}
$$

Now, let $x=\left\{x_{1}, \ldots, x_{N}\right\} \subset \mathscr{B}$ and $\theta=\left\{\theta_{1}, \ldots, \theta_{N}\right\} \subset \mathscr{F}$. We shall let $\mathscr{P}(\mathbb{A})$ be the Grassmann algebra $\mathscr{C}_{N}$ over the ring of polynomials in $x$ with coefficients in $\mathbb{A}$. Note that $\mathscr{P}(\mathbb{A})$ can simply be considered as the ring of polynomials in the variables $x$ and $\theta$ over $\mathbb{A}$.

It is obvious that $\mathscr{P}$ is bi-graded with respect to the bosonic and fermionic degrees, that is,

$$
\mathscr{P}=\bigoplus_{n, m \geq 0} \mathscr{P}_{(n \mid m)},
$$

where $\mathscr{P}_{(n \mid m)}$ is the finite dimensional module made out of all homogeneous polynomials $f(x, \theta)$ with degrees $n$ and $m$ in $x$ and $\theta$, respectively. Every polynomial $f(x, \theta)$ in $\mathscr{P}$ also possesses a bosonic and a fermionic part, i.e., $f(x, \theta)={ }^{0} f(x, \theta)+{ }^{1} f(x, \theta)$ where ${ }^{0} f(x, \theta) \in \mathscr{B}$ and ${ }^{1} f(x, \theta) \in \mathscr{F}$. We have that ${ }^{0} f(x, \theta)$ consists of the monomials of $f$ with an even degree in $\theta$ while ${ }^{1} f(x, \theta)$ consists of those monomials with an odd degree in $\theta$. Purely fermionic polynomials (i.e., elements of $\mathscr{P}_{(n \mid m)}$ with $m$ odd) have some nice properties. As an example, consider the following proposition that shall be useful in the subsequent sections.

Proposition 11. Let $\tilde{f}=\left\{\tilde{f}_{0}, \tilde{f}_{1}, \ldots\right\}$ and $\tilde{g}=\left\{\tilde{g}_{0}, \tilde{g}_{1}, \ldots\right\}$ be two sequences of fermionic polynomials parametrized by non-negative integers. Let also

$$
\tilde{f}_{\mu}:=\tilde{f}_{\mu_{1}} \tilde{f}_{\mu_{2}} \ldots \quad \text { and } \quad \tilde{g}_{\mu}:=\tilde{g}_{\mu_{1}} \tilde{g}_{\mu_{2}} \ldots
$$


where $\mu$ belongs to $\operatorname{Par}_{a}(n)$, the set of partitions of $n$ with strictly decreasing parts. Then

$$
\exp \left[\sum_{n=0}^{N-1} \tilde{f}_{n} \tilde{g}_{n}\right]=\sum_{n=0}^{N(N-1) / 2} \sum_{\mu \in \operatorname{Par}_{a}(n)} \overleftarrow{\widetilde{f}_{\mu}} \overrightarrow{\tilde{g}_{\mu}}
$$

Proof: Due to the fermionic character of $\tilde{f}$ and $\tilde{g}\left(\tilde{f}^{2}=\tilde{g}^{2}=0\right.$ for instance), we have

$$
\begin{aligned}
\exp \left[\sum_{n=0}^{N-1} \tilde{f}_{n} \tilde{g}_{n}\right] & =\prod_{0 \leq n \leq N-1}\left(1+\tilde{f}_{n} \tilde{g}_{n}\right) \\
& =1+\sum_{0 \leq n \leq N-1} \tilde{f}_{n} \tilde{g}_{n}+\sum_{0 \leq m<n \leq N-1} \tilde{f}_{m} \tilde{g}_{m} \tilde{f}_{n} \tilde{g}_{n}+\cdots
\end{aligned}
$$

Since every term in the last equality can be reordered by Lemma 10, the proof follows.

We finally define what we consider as symmetric polynomials in superspace. The algebra of symmetric superpolynomials over the $\operatorname{ring} \mathbb{A}$, denoted by $\mathscr{P} S_{N}(\mathbb{A})$ or by $\mathbb{A}\left[x_{1}, \ldots, x_{N}, \theta_{1}, \ldots, \theta_{N}\right]^{S_{N}}$, is a subalgebra of $\mathscr{P}$. As mentioned in the introduction, $\mathscr{P} S_{N}$ is made out of all $f(x, \theta) \in \mathscr{P}$ invariant under the diagonal action of the symmetric group $S_{N}$ on the two sets of variables.

To be more explicit, we introduce $K_{i j}$ and $\kappa_{i j}$, two distinct polynomial realizations of the transposition $(i, j) \in S_{N}$ :

$$
K_{i j} f\left(x_{i}, x_{j}, \theta_{i}, \theta_{j}\right)=f\left(x_{j}, x_{i}, \theta_{i}, \theta_{j}\right), \quad \kappa_{i j} f\left(x_{i}, x_{j}, \theta_{i}, \theta_{j}\right)=f\left(x_{i}, x_{j}, \theta_{j}, \theta_{i}\right),
$$

for all $f \in \mathscr{P}$. Since every permutation is generated by products of elementary transpositions $(i, i+1) \in S_{N}$, we can define symmetric superpolynomials as follows.

Definition 12. A polynomial $f(x, \theta) \in \mathscr{P}$ is symmetric if and only if

$$
\mathcal{K}_{i, i+1} f(x, \theta)=f(x, \theta) \quad \text { where } \quad \mathcal{K}_{i, i+1}:=\kappa_{i, i+1} K_{i, i+1}
$$

for all $i \in\{1,2, \ldots, N-1\}$.

Since every monomial $\theta_{J}=\theta_{j_{1}} \ldots \theta_{j_{m}}$ is completely antisymmetric,we have the following result.

Lemma 12. Let $f(x, \theta) \in \mathscr{P}$ be expressed as:

$$
f(x, \theta)=\sum_{m \geq 0} \sum_{1 \leq j_{1}<\cdots<j_{m} \leq N} f^{j_{1}, \ldots, j_{m}}(x) \theta_{j_{1}} \ldots \theta_{j_{m}} .
$$


If $f(x, \theta)$ is symmetric, then each polynomial $f^{j_{1}, \ldots, j_{m}}(x)$ is completely antisymmetric in the set of variables $y:=\left\{x_{j_{1}}, \ldots, x_{j_{m}}\right\}$ and completely symmetric in the set of variables $x \backslash y$.

Remark 14. The symmetric superpolynomials are completely different from the "supersymmetric polynomials" previously considered in the literature. Recall that what is called a supersymmetric polynomial (see e.g., [18]) is first of all a doubly symmetric polynomial in two distinct sets of ordinary (commuting) variables $x_{1}, \ldots x_{m}$ and $y_{1}, \ldots, y_{n}$, i.e., invariant under independent permutations of the $x_{i}$ 's and the $y_{i}$ 's. It is said to be supersymmetric if, in addition, it satisfies the following cancelation condition: by substituting $x_{1}=t$ and $y_{1}=t$, the polynomial becomes independent of $t$. An example of a generating function for such polynomials is

$$
\prod_{i=1}^{m}\left(1-q x_{i}\right) \prod_{j=1}^{n}\left(1-q y_{j}\right)^{-1}=\sum_{r \geq 0} p_{(r)}(x, y) q^{r}
$$

This generating function is known to appear in the context of classical Lie superalgebras (as a superdeterminant) [12]. Actually most of the work on supersymmetric polynomials is motivated by its connection with superalgebras. For an example of such an early work, see [11]. More precisions and references are also available in $[1,15]$. The key differences between these supersymmetric polynomials and our symmetric superpolynomials should be clear. In our case, we symmetrize two sets of variables with respect to the diagonal action of the symmetric group, with one of the two sets being made out of Grassmannian variables.

\subsection{Geometric interpretation of polynomials in superspace}

Symmetric functions can be interpreted as symmetric 0-forms $f$ acting on a manifold: $K_{i j} f(x)=f(x)$ where $x$ is a local coordinate system. Similarly, symmetric superfunctions in the $p$-fermion sector can be reinterpreted as symmetric $p$-forms $f^{p}$ acting on the same manifold: $\mathcal{K}_{i j} f^{p}(x)=f^{p}(x)$. Thus, the set of all symmetric superfunctions is in correspondence with the completely symmetric de Rham complex. This geometric point of view is briefly explained in this subsection. (Note that none of our results relies on this observation.)

We consider a Riemannian manifold $\mathcal{M}$ of dimension $N$ with metric $g_{i j}$ and let $x=\left\{x^{1}, \ldots, x^{N}\right\}$ denote a coordinate system on a given subset of $\mathcal{M}$. On the tangent bundle, we choose an orthonormal coordinate frame $\left\{\partial_{1}, \ldots, \partial_{N}\right\}$. As usual, $\left\{d x^{1}, \ldots, d x^{N}\right\}$ denotes the dual basis that belongs to the cotangent bundle, i.e., $d x^{i}\left(\partial_{x^{j}}\right)=\delta_{j}^{i}$. The set of all $p$-form fields on $\mathcal{M}$ is a vector space denoted by $\wedge^{p}$. Each $p$-form can be written as

$$
\alpha^{p}(x)=\sum_{1 \leq j_{1}<\cdots<j_{p} \leq N} \alpha_{j_{1}, \ldots, j_{p}}(x) d x^{j_{1}} \wedge \cdots \wedge d x^{j_{p}},
$$


where the exterior (wedge) product is antisymmetric: $d x^{i} \wedge d x^{j}=-d x^{j} \wedge d x^{i}$. Let $d$ be the exterior differentiation on forms, whose action is

$$
d \alpha^{p}(x)=\sum_{1 \leq k, j_{1}, \ldots, j_{p} \leq N}\left[\partial_{x^{k}} \alpha_{j_{1}, \ldots, j_{p}}(x)\right] d x^{k} \wedge d x^{j_{1}} \wedge \cdots \wedge d x^{j_{p}} .
$$

This operation is used to define the de Rham complex of $\mathcal{M}$ :

$$
0 \longrightarrow \mathbb{R} \longrightarrow \bigwedge^{0} \stackrel{d}{\longrightarrow} \bigwedge^{1} \stackrel{d}{\longrightarrow} \cdots \stackrel{d}{\longrightarrow} \bigwedge^{N} \stackrel{d}{\longrightarrow} 0
$$

In order to represent our Grassmannian variables $\theta^{j}$ and $\theta^{j \dagger}$ in terms of forms, we introduce the two operators $\hat{e}_{d x^{j}}$ and $\hat{\mathrm{i}}_{\partial_{x_{k}}}$, where $\hat{e}_{\alpha}$ and $\hat{\mathrm{i}}_{v}$ respectively stand for the left exterior product by the form $\alpha$ and the interior product (contraction) with respect to the vector field $v$. These operators satisfy a Clifford (fermionic) algebra

$$
\left\{\hat{e}_{d x^{i}}, \hat{\mathrm{1}}_{\partial_{x_{k}}}\right\}=\delta_{k}^{i} \quad \text { and } \quad\left\{\hat{e}_{d x^{i}}, \hat{e}_{d x^{j}}\right\}=0=\left\{\hat{\mathrm{i}}_{\partial_{x_{j}}}, \hat{\mathrm{1}}_{\partial_{x_{k}}}\right\}
$$

This implies that the $\theta^{j}$ 's and $\theta^{j \dagger}$, as operators, can be realized as follows:

$$
\theta^{j} \sim \hat{e}_{d x^{j}} \text { and } \theta^{j \dagger} \sim g^{j k} \hat{\mathbf{1}}_{\partial_{x_{k}}}
$$

that is,

$$
d x^{j} \sim \theta^{j}(1) \quad \text { and } \quad \theta^{j \dagger} \sim g^{j k} \partial_{\theta^{k}} \quad \text { where } \quad \partial_{\theta^{k}}:=\frac{\partial}{\partial \theta^{k}} .
$$

Note that introducing the Grassmannian variables as operators is needed to enforce the wedge product of the forms $d x_{j}$. Moreover, if $\alpha^{p}$ is a generic $p$-form field and $\hat{\pi}: \bigwedge^{p} \rightarrow \bigwedge^{p}$ is the operator defined by

$$
\hat{\pi}_{p}:=\theta^{j} \partial_{\theta^{j}}=\theta^{j} g_{j k} \theta^{k \dagger} \Longrightarrow \hat{\pi}_{p} \alpha^{p}=p \alpha^{p},
$$

then $\hat{\Pi}_{p}:=(-1)^{\hat{\pi}_{p}}$ is involutive. (Manifestly, $\hat{\Pi}_{p}=\hat{\Pi}$, the parity operator introduced previously.) This involution is also an isometry in the Hilbert space scalar product. The operator $\hat{\Pi}_{p}$ induces a natural $\mathbb{Z}_{2}$ grading in the de Rham complex.

The construction of the symmetric de Rham complex is obtained as follows. We make a change of coordinates: $x \rightarrow f(x)$, where $f=\left\{f^{n}\right\}:=\left\{f^{1}, \ldots, f^{N}\right\}$ is an $N$-tuple of symmetric and independent functions of $x$. For instance, $f^{n}$ could be an elementary symmetric function $e_{n}$, a complete symmetric function $h_{n}$, or a power sum $p_{n}$ (see Section 3). This implies a change of basis in the cotangent bundle: $d x \rightarrow d f(x)$. Explicitly,

$$
d f^{n}=\sum_{i}\left(\partial_{i} f^{n}\right)(x) d x_{i} \sim \tilde{f}^{n}=\sum_{i}\left(\partial_{i} f^{n}\right)(x) \theta_{i}
$$

In other words, $d f$ is a new set of "fermionic" variables invariant under any permutation of the $x_{j}$ 's. 
These remarks explicitly show that symmetric polynomials in superspace can be interpreted as symmetric differential forms. We stress that the diagonal action of the symmetric group $S_{N}$ comes naturally in the geometric perspective. Note finally that for an Euclidian superspace (relevant to our context), the position (upper or lower) of the indices does not matter.

\subsection{Monomial basis}

The monomial symmetric functions in superspace, denoted by $m_{\Lambda}=m_{\Lambda}(x, \theta)$, are the superanalog of the monomial symmetric functions.

Definition 15 ([4]). To each $\Lambda \in \operatorname{SPar}(n \mid m)$, we associate the monomial symmetric function

$$
m_{\Lambda}=\sum_{\sigma \in S_{N}}^{\prime} \theta^{\sigma(1, \ldots, m)} x^{\sigma(\Lambda)}
$$

where the prime indicates that the summation is restricted to distinct terms, and where

$$
x^{\sigma(\Lambda)}=x_{1}^{\Lambda_{\sigma(1)}} \ldots x_{m}^{\Lambda_{\sigma(m)}} x_{m+1}^{\Lambda_{\sigma(m+1)}} \ldots x_{N}^{\Lambda_{\sigma(N)}} \quad \text { and } \quad \theta^{\sigma(1, \ldots, m)}=\theta_{\sigma(1)} \ldots \theta_{\sigma(m)} .
$$

Obviously, the previous definition can be replaced by the following:

$$
m_{\Lambda}=\frac{1}{n_{\Lambda} !} \sum_{\sigma \in S_{N}} \mathcal{K}_{\sigma}\left(\theta_{1} \ldots \theta_{m} x^{\Lambda}\right)
$$

with

$$
n_{\Lambda} !=n_{\Lambda^{s}} !:=n_{\Lambda^{s}}(0) ! n_{\Lambda^{s}}(1) ! n_{\Lambda^{s}}(2) ! \ldots,
$$

where $n_{\Lambda^{s}}(i)$ indicates the number of $i$ 's in $\Lambda^{s}$, the symmetric part of $\Lambda=\left(\Lambda^{a} ; \Lambda^{s}\right)$. Moreover, $\mathcal{K}_{\sigma}$ stands for $\mathcal{K}_{i_{1}, i_{1}+1} \ldots \mathcal{K}_{i_{n}, i_{n}+1}$ when the element $\sigma$ of the symmetric group $S_{N}$ is written in terms of elementary transpositions, i.e., $\sigma=\sigma_{i_{1}} \ldots \sigma_{i_{n}}$. Notice that the monomial symmetric function $m_{\Lambda}$, with $\Lambda \vdash(n \mid m)$, belongs to $\mathscr{P}_{(n \mid m)}(\mathbb{Z})$, the space of superpolynomials of degree $(n \mid m)$ with integer coefficients.

Theorem 16. The set $\left\{m_{\Lambda}\right\}_{\Lambda \vdash(n \mid m)}:=\left\{m_{\Lambda}: \Lambda \in \operatorname{SPar}(n \mid m)\right\}$ is a basis of $\mathscr{P}_{(n \mid m)}^{S_{N}}(\mathbb{Z})$.

Proof: Each polynomial $f(x, \theta)$ of degree $(n \mid m)$, with $N$ variables and with integer coefficients, can be expressed as a sum of monomials of the type $\theta_{j_{1}} \ldots \theta_{j_{m}} x^{\mu}$, with coefficient $a_{\mu}^{j_{1}, \ldots, j_{m}} \in \mathbb{Z}$, and where $\mu$ is a composition of $N$. Let $\Omega^{a}$ be the reordering of the entries $\left(\mu_{j_{1}}, \ldots, \mu_{j_{m}}\right)$, and let $\Omega^{s}$ be the reordering of the remaining entries of $\mu$. Because the polynomial $f(x, \theta)$ is also symmetric, $f(x, \theta)$ is by definition Springer 
invariant under the action of $\mathcal{K}_{\sigma}$, for any $\sigma \in S_{N}$. Therefore, $a_{\mu}^{j_{1}, \ldots, j_{m}}$ must be equal, up to a sign, to the coefficient $a_{\Omega}^{1,2, \ldots, m}$ of $\theta_{1} \ldots \theta_{m} x^{\Omega}$ in $f(x, \theta)$, where $\Omega=\left(\Omega^{a} ; \Omega^{s}\right)$. Note that from Lemma $12, \Omega^{a}$ needs to have distinct parts, which means that $\Omega$ is a superpartition. This gives that $f(x, \theta)-a_{\Omega}^{1,2, \ldots, m} m_{\Omega}$ does not contain any monomial that is also a monomial of $m_{\Omega}$, since otherwise it would need by symmetry to contain the monomial $\theta_{1} \ldots \theta_{m} x^{\Omega}$.

Now, consider any total order on superpartitions, and let $\Lambda$ be the highest superpartition in this order such that there is a monomial of $m_{\Lambda}$ appearing in $f(x, \theta)$. By the previous argument, $f(x, \theta)-a_{\Lambda}^{1, \ldots, m} m_{\Lambda}$ is a symmetric superpolynomial such that no monomial belonging to $m_{\Lambda}$ appears in its expansion. Since no monomial of $m_{\Lambda}$ appears in any other monomial of $m_{\Omega}$, for $\Omega \neq \Lambda$, the proof follows by induction.

Corollary 17. The set $\left\{m_{\Lambda}\right\}_{\Lambda}:=\left\{m_{\Lambda}: \Lambda \in \operatorname{SPar}\right\}$ is a basis of $\mathscr{P}^{S_{N}}(\mathbb{Z})$.

This corollary implies that $\mathscr{P}^{S_{N}}(\mathbb{Z})$ could also be defined as the free $\mathbb{Z}$-module spanned by the set of monomial symmetric functions in superspace.

To end this section, we give a formula for the expansion coefficients of the product of two monomial symmetric functions in terms of monomial symmetric functions. In this kind of calculation, the standard counting of combinatorial objects is affected by signs resulting from the reordering of fermionic variables.

Definition 18. Let $\Lambda \in \operatorname{SPar}(n \mid m), \Omega \in \operatorname{SPar}\left(n^{\prime} \mid m^{\prime}\right)$ and $\Gamma \in \operatorname{SPar}\left(n+n^{\prime} \mid m+m^{\prime}\right)$. In each box or circle of $D[\Lambda]$, we write a letter $a$. In its $i$-th circle (the one corresponding to $\Lambda_{i}$ ), we add the label $i$ to the letter $a$. We do the same process for $D[\Omega]$ replacing $a$ by $b$. We then define $\mathcal{T}[\Lambda, \Omega ; \Gamma]$ to be the set of distinct fillings of $D[\Gamma]$ with the letters of $D[\Lambda]$ and $D[\Omega]$ obeying the following rules:

(1) the circles of $D[\Gamma]$ can only be filled with labeled letters (an $a_{i}$ or a $b_{i}$ );

(2) each row of the filling of $D[\Lambda]$ is reproduced in a single and distinct row of the filling of $D[\Gamma]$; in other words, rows of $D[\Lambda]$ cannot be split and two rows of $D[\Lambda]$ cannot be put within a single row of $D[\Gamma]$

(3) rule 2 also holds when $D[\Lambda]$ is replaced by $D[\Omega]$;

(4) in each row, the unlabeled $a$ 's appear to the left of the unlabeled $b$ 's.

For instance, there are three possible fillings of $\left(2,1,0 ; 1^{3}\right)$ with $(1,0 ; 1)$ and $\left(0 ; 2,1^{2}\right)$ :

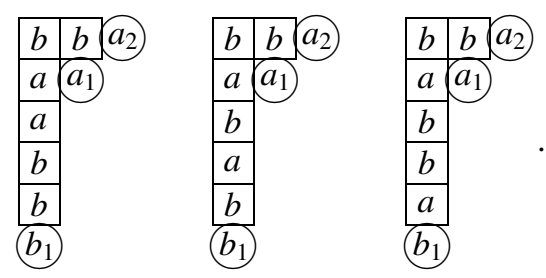


There are also three possible fillings of $\left(3,1,0 ; 1^{2}\right)$ with $(1,0 ; 1)$ and $\left(0 ; 2,1^{2}\right)$ :
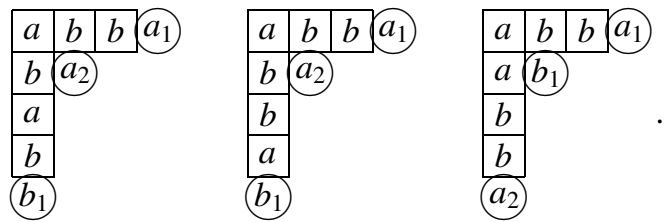

Definition 19. Let $T \in \mathcal{T}[\Lambda, \Omega ; \Gamma]$, with $\underline{\bar{\Lambda}}=m$ and $\underline{\bar{\Omega}}=m^{\prime}$. The weight of $T$, denoted by $\hat{w}[T]$, corresponds to the sign of the permutation needed to reorder the content of the circles in the filling of $D[\Gamma]$ so that from top to bottom they read as $a_{1} \ldots a_{m} b_{1} \ldots b_{m^{\prime}}$.

In the example (2.41), each term has weight -1 (odd parity). The oddness of these fillings comes from the transposition that is needed to reorder $a_{1}$ and $a_{2}$. In the second example (2.4), the two first fillings are even while the last filling is odd due to the needed transposition of $a_{2}$ and $b_{1}$. As we shall see in the next proposition, the two previous sets lead respectively to the coefficients of $m_{\left(2,1,0 ; 1^{3}\right)}$ and $m_{\left(3,1,0 ; 1^{2}\right)}$ in the product of $m_{(1,0 ; 1)}$ and $m_{\left(0 ; 2,1^{2}\right)}$, that is,

$$
m_{(1,0 ; 1)} m_{\left(0 ; 2,1^{2}\right)}=\underbrace{(-3)}_{-1-1-1} \times m_{\left(2,1,0 ; 1^{3}\right)}+\underbrace{(1)}_{+1+1-1} \times m_{\left(3,1,0 ; 1^{2}\right)}+\text { other terms. }
$$

Proposition 20. Let $m_{\Lambda}$ and $m_{\Omega}$ be any two monomial symmetric functions in superspace. Then

$$
m_{\Lambda} m_{\Omega}=\sum_{\Gamma \in \mathrm{SPar}} N_{\Lambda, \Omega}^{\Gamma} m_{\Gamma}
$$

where the integer $N_{\Lambda, \Omega}^{\Gamma}=(-1)^{\bar{\Lambda}} \cdot \underline{\bar{\Omega}} N_{\Omega, \Lambda}^{\Gamma}$ is given by

$$
N_{\Lambda, \Omega}^{\Gamma}:=\sum_{T \in \mathcal{T}[\Lambda, \Omega ; \Gamma]} \hat{w}[T]
$$

Proof: From the symmetry property in Definition 15 , the coefficient $N_{\Lambda, \Omega}^{\Gamma}$ is simply given by the coefficient of $\theta_{\{1, \ldots, m+p\}} x^{\Gamma}$ in $m_{\Lambda} m_{\Omega}$. The terms contributing to this coefficient correspond to all distinct permutations $\sigma$ and $w$ of the entries of $\Lambda$ and $\Omega$ respectively such that

$$
\Gamma=\left(\Lambda_{\sigma(1)}+\Omega_{w(1)}, \ldots, \Lambda_{\sigma(N)}+\Omega_{w(N)}\right),
$$

where the entries of $\Lambda^{a}$ and $\Omega^{a}$ are distributed among the first $m+p$ entries (no two in the same position). But this set is easily seen to be in correspondence with the fillings in $\mathcal{T}[\Lambda, \Omega ; \Gamma]$ when realizing that labeled letters simply give the positions of the fermions in $C[\Gamma]$ (the circled version of $\Gamma$ ). The only remaining problem is thus 祭 Springer 
the ordering of the fermions. In (2.46), from the definition of monomial symmetric functions, the sign of the contribution is equal to the sign of the permutation needed to reorder the fermionic entries of $\Lambda^{a}$ and $\Omega^{a}$ that are distributed among the first $m+p$ entries so that they correspond to $\left(\Lambda^{a}, \Omega^{a}\right)$. But this is simply the sign of the permutation that reorders the circled entries in the corresponding filling of $D[\Gamma]$ such that they read as $a_{1} \ldots a_{m} b_{1} \ldots b_{p}$.

\section{Generating functions and multiplicative bases}

In the theory of symmetric functions, the number of variables is usually irrelevant, and can be set for convenience to be equal to infinity. In a similar way, we shall consider from now on that, unless otherwise specified, the number of $x$ and $\theta$ variables is infinite, and denote the ring of symmetric superfunctions as $\mathscr{P} S_{\infty}$.

\subsection{Elementary symmetric functions}

Let $J=\left\{j_{1}, \ldots, j_{r}\right\}$ with $1 \leq j_{1}<j_{2}<j_{3} \cdots$ and let $\# J:=\operatorname{Card} J$. The $n$-th bosonic and fermionic elementary symmetric functions, for $n \geq 1$, are defined respectively by

$$
e_{n}:=\sum_{J ; \# J=n} x_{j_{1}} \ldots x_{j_{n}} \quad \text { and } \quad \tilde{e}_{n}:=\sum_{i \geq 1} \sum_{\substack{J ; \# J=n \\ i \notin J}} \theta_{i} x_{j_{1}} \ldots x_{j_{n}}
$$

In addition, we impose

$$
e_{0}=1 \quad \text { and } \quad \tilde{e}_{0}=\sum_{i} \theta_{i}
$$

So, in terms of monomials, we have

$$
e_{n}=m_{\left(1^{n}\right)}, \quad \tilde{e}_{n}=m_{\left(0 ; 1^{n}\right)}
$$

We introduce two parameters: $t \in \mathscr{B}$ and $\tau \in \mathscr{F}$. It is easy to verify that the generating function for the elementary symmetric functions is

$$
E(t, \tau):=\sum_{n=0}^{\infty} t^{n}\left(e_{n}+\tau \tilde{e}_{n}\right)=\prod_{i=1}^{\infty}\left(1+t x_{i}+\tau \theta_{i}\right) .
$$

Actually, to go from the usual generating function $E(t):=E(t, 0)$ to the new one, one simply replaces $x_{i} \rightarrow x_{i}+\tau^{\prime} \theta_{i}$ and redefines $\tau^{\prime}=\tau / t$, an operation that makes manifest the invariance of $E(t, \tau)$ under the simultaneous interchange of the $x_{i}$ 's and the $\theta_{i}$ 's.

From an analytic point of view, the fermionic elementary symmetric functions are obtained by exterior differentiation:

$$
\tilde{e}_{n-1}(x, \theta) \sim \tilde{e}_{n-1}(x, d x)=d e_{n}(x),
$$


for all $n \geq 1$. How can we explain that the generating function (3.4) leads precisely to the fermionic elementary functions that are obtained by the action of the exterior derivative of the elementary symmetric function? The rationale for this feature turns out to be rather simple. Indeed, let $\tau:=t d t$ and define $D$ to act on a function $f(x, t)$ as a tensor-product derivative:

$$
D f:=d t \wedge d f .
$$

In consequence, we formally have

$$
\left(1+t x_{i}+\tau \theta_{i}\right) \sim(1+D)\left(1+t x_{i}\right) \text { and } E(t, \tau) \sim(1+D) E(t)
$$

which is the desired link.

In order to obtain a new basis of the symmetric superpolynomial algebra, we associate, to each superpartition $\Lambda=\left(\Lambda_{1}, \ldots, \Lambda_{m} ; \Lambda_{m+1}, \ldots, \Lambda_{\ell}\right)$ of $(n \mid m)$, a polynomial $e_{\Lambda} \in \mathscr{P} \mathscr{P}_{(n \mid m)}^{S_{\infty}}$ defined by

$$
e_{\Lambda}:=\prod_{i=1}^{m} \tilde{e}_{\Lambda_{i}} \prod_{j=m+1}^{\ell} e_{\Lambda_{j}},
$$

Note that the product of anticommutative quantities is always done from left to right: $\prod_{i=1}^{N} F_{i}:=F_{1} F_{2} \ldots F_{N}$. We stress that the ordering matters in the fermionic sector since for instance

$$
e_{(3,0 ; 4,1)}=\tilde{e}_{3} \tilde{e}_{0} e_{4} e_{1}=-\tilde{e}_{0} \tilde{e}_{3} e_{4} e_{1}
$$

Theorem 21. Let $\Lambda$ be a superpartition of $(n \mid m)$ and $\Lambda^{\prime}$ its conjugate. Then

$$
\overleftarrow{e_{\Lambda}}=\tilde{e}_{\Lambda_{m}} \ldots \tilde{e}_{\Lambda_{1}} e_{\Lambda_{m+1}} \ldots e_{\Lambda_{N}}=m_{\Lambda^{\prime}}+\sum_{\Omega<\Lambda^{\prime}} N_{\Lambda}^{\Omega} m_{\Omega}
$$

where $N_{\Lambda}^{\Omega}$ is an integer. Hence, $\left\{e_{\Lambda}: \Lambda \vdash(n \mid m)\right\}$ is a basis of $\mathscr{P}_{(n \mid m)} S_{\infty}(\mathbb{Z})$.

Proof: We first observe that $\overleftarrow{e_{\Lambda}}=(-1)^{m(m-1) / 2} e_{C[\Lambda]}$, where $C[\Lambda]$ denotes as usual the partition $\Lambda^{*}$ in which fermionic parts of $\Lambda$ are identified by a circle. Then, assuming that we work in $N$ variables, the monomials $\theta_{J} x^{\nu}$ that appear in the expansion of $e_{C[\Lambda]}$ are in correspondence with the fillings of $D\left[\Lambda^{\prime}\right]$ with the letters $1, \ldots, N$ such that:

(1) the non-circled entries in the filling of $D\left[\Lambda^{\prime}\right]$ increase when going down in a column;

(2) if a column contains a circle, then the entry that fills the circle cannot appear anywhere else in the column.

The correspondence follows because the reading of the $i$-th column corresponds to one monomial of $e_{\Lambda_{i}}$ (or $\tilde{e}_{\Lambda_{i}}$ ). To be more specific, if the reading of the column is 祭 Springer 
$j_{1}, \ldots, j_{\Lambda_{i}}$ (with a possible extra letter $a$ ), it corresponds to the monomial $x_{j_{1}} \ldots x_{j_{\Lambda_{i}}}$ ( or $\theta_{a} x_{j_{1}} \ldots x_{j_{\Lambda_{i}}}$ ). The first condition ensures that we do not count the permutations of $x_{j_{1}} \ldots x_{j_{\Lambda_{i}}}$ as distinct monomials. The second one ensures that in the fermionic case, the index of $\theta_{a}$ is distinct from the index of the variables $x_{j_{1}}, \ldots, x_{j_{\Lambda_{i}}}$.

Now, to obtain the coefficient $N_{\Lambda}^{\Omega}$, it suffices to compute the coefficient of $\theta_{C[\Omega]} x^{C[\Omega]}$ in $e_{C[\Lambda]}$, where $\theta_{C[\Omega]}$ represents the product of the fermionic entries of $C[\Omega] \mathrm{read}$ from left to right. Note that this coefficient has the same sign as $\theta_{\{1, \ldots, m\}} x^{\Omega}$ in $m_{\Omega}$ and there is thus no need to compensate by a sign factor. The monomials contributing to $N_{\Lambda}^{\Omega}$ are therefore fillings of $D\left[\Lambda^{\prime}\right]$ (obeying the two conditions given above) with the letter $i$ appearing $C[\Omega]_{i}$ times in non-circled cells with one additional time in a circled cell if $C[\Omega]_{i}$ is fermionic. We will call the set of such fillings $\mathcal{T}^{(e)}\left[\Omega ; \Lambda^{\prime}\right]$.

Finally, given a filling $T \in \mathcal{T}^{(e)}\left[\Omega ; \Lambda^{\prime}\right]$, we read the content of the circles from top to bottom and obtain a word $a_{1} \ldots a_{m}$. The sign of the permutation needed to reorder this word such that it be increasing gives the weight associated to the filling $T$, denoted this time $\bar{w}[T]$. The weight of $T$ is the sign needed to reorder the monomial associated to $T$ so that it corresponds to $\theta_{C[\Omega]} x^{C[\Omega]}$ up to a factor $(-1)^{m(m-1) / 2}$. This is because to coincide with the product in $e_{C[\Lambda]}$ being done columnwise, we would have to read from bottom to top. Reading from top to bottom provides the $(-1)^{m(m-1) / 2}$ factor needed to obtain the coefficient in $\overleftarrow{e_{\Lambda}}$ instead of in $e_{C[\Lambda]}$. We thus have

$$
N_{\Lambda}^{\Omega}=\sum_{T \in \mathcal{T}^{(e)}\left[\Omega ; \Lambda^{\prime}\right]} \bar{w}[T]
$$

We now use this equation to prove the theorem.

First, it is easy to convince ourselves that there is only one element in $\mathcal{T}^{(e)}\left[\Lambda^{\prime} ; \Lambda^{\prime}\right]$ and that it has a positive weight. Because the rows of $D\left[\Lambda^{\prime}\right]$ and $C\left[\Lambda^{\prime}\right]$ coincide, for the filling to have increasing rows, we have no choice but to put the $C\left[\Lambda^{\prime}\right]_{i}$ letters $i$ in the $i$-th row of $D\left[\Lambda^{\prime}\right]$. In the case when $C\left[\Lambda^{\prime}\right]_{i}$ is fermionic, the extra $i$ has no choice but to go in the circle in row $i$ of $D\left[\Lambda^{\prime}\right]$ for no two $i$ 's to be in a same column. For example, given $\Lambda^{\prime}=(3,1 ; 2,1)$ filling $D\left[\Lambda^{\prime}\right]$ with the letters of $C\left[\Lambda^{\prime}\right]$ leads to:

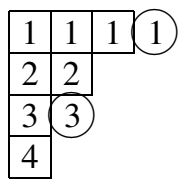

in
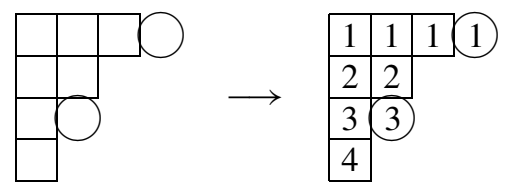

This explains the first term in (3.10).

Second, let $\omega=\Omega^{*}$ and $\lambda=\Lambda^{*}$. If $\Omega \nless_{S} \Lambda^{\prime}$, a filling of $\Omega$ by $\Lambda$ is obviously impossible because we would need to be able to obtain in particular (forgetting about the circles) a filling of the type $\mathcal{T}^{(e)}\left[\omega ; \lambda^{\prime}\right]$, which would contradict the well known fact that the theorem holds in the zero-fermion case.

Finally, we suppose that $\Lambda^{*}=\Omega^{*}$ and $\operatorname{sh}(D[\Omega]) \nless \operatorname{sh}\left(D\left[\Lambda^{\prime}\right]\right.$ ) (see Corollary 7). Again, this is impossible because we would need to be able to obtain in particular (considering the circles as usual cells and reordering the columns) a filling of the type $\mathcal{T}^{(e)}\left[\operatorname{sh}(D[\Omega]) ; \operatorname{sh}\left(D\left[\Lambda^{\prime}\right]\right)\right]$, which would contradict the well known fact that the theorem holds in the zero-fermion case. 
Note that for the various examples that we have worked out, the coefficients $N_{\Lambda}^{\Omega}$ happened to be non-negative. So we may surmise that a stronger version of the theorem, where $N_{\Lambda}^{\Omega}$ is a non-negative integer, holds.

The linear independence of the $e_{\Lambda}$ 's in $\mathscr{P}^{S_{N}}$ implies that the first $N$ bosonic and fermionic elementary functions are algebraically independent over $\mathbb{Z}$. Symbolically,

$$
\mathbb{Z}\left[x_{1}, \ldots, x_{N}, \theta_{1}, \ldots, \theta_{N}\right]^{S_{N}} \equiv \mathbb{Z}\left[e_{1}, \ldots, e_{N}, \tilde{e}_{0}, \ldots \tilde{e}_{N-1}\right]
$$

which can be interpreted as the fundamental theorem of symmetric polynomials in superspace.

\subsection{Complete symmetric functions and involution}

The $n$-th bosonic and fermionic complete symmetric functions are given respectively by

$$
h_{n}:=\sum_{\lambda \vdash n} m_{\lambda} \quad \text { and } \quad \tilde{h}_{n}:=\sum_{\Lambda \vdash(n \mid 1)}\left(\Lambda_{1}+1\right) m_{\Lambda},
$$

From the explicit form of $h_{n}(x)$, namely, $\sum_{1 \leq i_{1} \leq i_{2} \leq \cdots \leq i_{n}} x_{i_{1}} \ldots x_{i_{n}}$, we see that its fermionic partner is again generated by the action of $d$ in the form-representation:

$$
\tilde{h}_{n-1}(x, \theta) \sim \tilde{h}_{n-1}(x, d x)=d h_{n}(x) \text { for all } n \geq 1 .
$$

The generating function for complete symmetric polynomials is

$$
H(t, \tau):=\sum_{n=0}^{\infty} t^{n}\left(h_{n}+\tau \tilde{h}_{n}\right)=\prod_{i=1}^{\infty} \frac{1}{1-t x_{i}-\tau \theta_{i}} .
$$

To prove (3.16), one simply uses the inversion of even elements in the Grassmann algebra, which gives

$$
\frac{1}{1-t x_{i}-\tau \theta_{i}}=\sum_{n \geq 0}\left(t x_{i}+\tau \theta_{i}\right)^{n}=\sum_{n \geq 0}\left[\left(t x_{i}\right)^{n}+n \tau \theta_{i}\left(t x_{i}\right)^{n-1}\right] .
$$

From relations (3.4) and (3.16), we get

$$
H(t, \tau) E(-t,-\tau)=1
$$

By expanding the generating functions in terms of $e_{n}, \tilde{e}_{n}, h_{n}$ and $\tilde{h}_{n}$ in the last equation, we obtain recursion relations, of which the non-fermionic one is a well known formula.

Lemma 22. Let $n \geq 1$, then

$$
\sum_{r=0}^{n}(-1)^{r} e_{r} h_{n-r}=0
$$


Let $n \geq 0$, then

$$
\sum_{r=0}^{n}(-1)^{r}\left(e_{r} \tilde{h}_{n-r}-\tilde{e}_{r} h_{n-r}\right)=0
$$

Note that the second relation can be obtained from the first one by the action of $d$ (representing, as usual, $\theta_{i}$ as $d x_{i}$ ).

We consider a homomorphism $\hat{\omega}: \mathscr{P} S_{\infty}(\mathbb{Z}) \rightarrow \mathscr{P} S_{\infty}(\mathbb{Z})$ defined by the following relations:

$$
\hat{\omega}: e_{n} \longmapsto h_{n} \quad \text { and } \quad \tilde{e}_{n} \longmapsto \tilde{h}_{n}
$$

Theorem 23. The homomorphism $\hat{\omega}$ is an involution, i.e., $\hat{\omega}^{2}=1$. Equivalently, we have

$$
\hat{\omega}: h_{n} \longmapsto e_{n} \quad \text { and } \quad \tilde{h}_{n} \longmapsto \tilde{e}_{n}
$$

Proof: This comes from the application of transformation (3.21) to the recursions appearing in Lemma 22 followed by the comparison with the original recursions. Explicitly:

$$
0=\sum_{r=0}^{n}(-1)^{r} \hat{\omega}\left(e_{r}\right) \hat{\omega}\left(h_{n-r}\right)=(-1)^{n} \sum_{r=0}^{n}(-1)^{r} \hat{\omega}\left(h_{r}\right) h_{n-r},
$$

which implies $\hat{\omega}\left(h_{r}\right)=e_{r}$. Similarly, we have

$$
\begin{aligned}
0 & =\sum_{r=0}^{n}(-1)^{r}\left(\hat{\omega}\left(e_{r}\right) \hat{\omega}\left(\tilde{h}_{n-r}\right)-\hat{\omega}\left(\tilde{e}_{r}\right) \hat{\omega}\left(h_{n-r}\right)\right) \\
& =(-1)^{n-1} \sum_{r=0}^{n}(-1)^{r}\left(e_{r} \tilde{h}_{n-r}-\hat{\omega}\left(\tilde{h}_{r}\right) h_{n-r}\right),
\end{aligned}
$$

leading to $\hat{\omega}\left(\tilde{h}_{r}\right)=\tilde{e}_{r}$.

Now, let

$$
h_{\Lambda}:=\prod_{i=1}^{\bar{\Lambda}} \tilde{h}_{\Lambda_{i}} \prod_{j=\underline{\underline{\Lambda}}+1}^{\ell(\Lambda)} h_{\Lambda_{j}}
$$

Equation (3.21) and Theorem 23 immediately give a bijection between two sets of multiplicative polynomials:

$$
\hat{\omega}\left(e_{\Lambda}\right)=h_{\Lambda} \quad \text { and } \quad \hat{\omega}\left(h_{\Lambda}\right)=e_{\Lambda}
$$


We have thus obtained another $\mathbb{Z}$-basis for the algebra of symmetric superpolynomials.

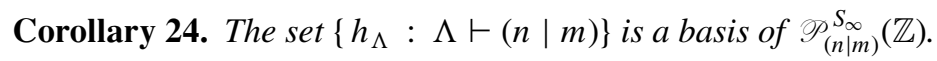

Finally, Lemma 22 allows us to write determinantal expressions for the elementary symmetric functions in terms of the complete symmetric functions and vice versa using the homomorphism $\hat{\omega}$.

Proposition 25. For $n \geq 1$, we have

$$
e_{n}=\left|\begin{array}{cccccc}
h_{1} & h_{2} & h_{3} & \cdots & h_{n-1} & h_{n} \\
1 & h_{1} & h_{2} & \cdots & h_{n-2} & h_{n-1} \\
0 & 1 & h_{1} & \cdots & h_{n-3} & h_{n-2} \\
0 & 0 & 1 & \ddots & h_{n-4} & h_{n-3} \\
\vdots & \vdots & \ddots & \ddots & \ddots & \vdots \\
0 & 0 & 0 & \cdots & 1 & h_{1}
\end{array}\right|
$$

For $n \geq 0$, we have

$\tilde{e}_{n}=\frac{1}{n !}\left|\begin{array}{cccccc}\tilde{h}_{0} & \tilde{h}_{1} & \tilde{h}_{2} & \cdots & \tilde{h}_{n-1} & \tilde{h}_{n} \\ n & (n+1) h_{1} & (n+2) h_{2} & \cdots & (2 n-1) h_{n-1} & 2 n h_{n} \\ 0 & n-1 & n h_{1} & \cdots & (2 n-3) h_{n-2} & (2 n-2) h_{n-1} \\ 0 & 0 & n-2 & \ddots & (2 n-5) h_{n-3} & (2 n-4) h_{n-2} \\ \vdots & \vdots & \ddots & \ddots & \ddots & \vdots \\ 0 & 0 & 0 & \cdots & 1 & 2 h_{1}\end{array}\right|$

Proof: The first relation is well known to be a simple application of Cramer's rule to the linear system coming from Lemma $22: \mathbf{h}=\mathbf{e} \mathbf{H}$, where

$$
\mathbf{h}^{\mathrm{t}}=\left(\begin{array}{c}
h_{1} \\
h_{2} \\
h_{3} \\
\vdots \\
h_{N}
\end{array}\right) \quad \mathbf{e}^{\mathrm{t}}=\left(\begin{array}{c}
e_{1} \\
e_{2} \\
e_{3} \\
\vdots \\
e_{N}
\end{array}\right) \quad \mathbf{H}=\left(\begin{array}{ccccc}
1 & h_{1} & h_{2} & h_{3} & \cdots \\
0 & -1 & -h_{1} & -h_{2} & \ldots \\
0 & 0 & 1 & h_{1} & \ddots \\
0 & 0 & 0 & -1 & \ddots \\
\vdots & \ddots & \ddots & \ddots & \ddots
\end{array}\right)
$$


To obtain the other determinant, we use the second formula of Lemma 22 to obtain the linear system:

$$
\tilde{\mathbf{h}} \overline{\mathbf{H}}=\tilde{\mathbf{e}} \mathbf{H},
$$

where $\mathbf{H}$ is as given above, and where

$$
\tilde{\mathbf{h}}^{\mathrm{t}}=\left(\begin{array}{c}
\tilde{h}_{0} \\
\tilde{h}_{1} \\
\tilde{h}_{2} \\
\vdots \\
\tilde{h}_{N-1}
\end{array}\right) \quad \tilde{\mathbf{e}}^{\mathrm{t}}=\left(\begin{array}{c}
\tilde{e}_{0} \\
\tilde{e}_{1} \\
\tilde{e}_{2} \\
\vdots \\
\tilde{e}_{N-1}
\end{array}\right) \quad \overline{\mathbf{H}}=\left(\begin{array}{rrrrr}
1 & -e_{1} & e_{2} & -e_{3} & \ldots \\
0 & 1 & -e_{1} & e_{2} & \ldots \\
0 & 0 & 1 & -e_{1} & \ddots \\
0 & 0 & 0 & 1 & \ddots \\
\vdots & \ddots & \ddots & \ddots & \ddots
\end{array}\right)
$$

Using the coadjoint formula for the inverse of a matrix, and the determinantal expression for $h_{n}$ obtained by applying the homomorphism $\hat{\omega}$ on the determinant of $e_{n}$ given above, it is not hard to see that the $(i, j)$-th component of the inverse of $\overline{\mathbf{H}}$ is simply $h_{j-i}$. We are thus led to the matrix relation:

$$
\tilde{\mathbf{h}}=\tilde{\mathbf{e}} \widetilde{\mathbf{H}}
$$

where

$$
\widetilde{\mathbf{H}}_{i, j}=(-1)^{i+1} \sum_{k \geq 0}^{i-j} h_{i-j-k} h_{k}=-\widetilde{\mathbf{H}}_{i+1, j+1}
$$

If we set $H_{i}=\sum_{k=0}^{i} h_{i-k} h_{k}$, the matrix $\widetilde{\mathbf{H}}$ can be expressed in a convenient form as

$$
\widetilde{\mathbf{H}}=\left(\begin{array}{rrrrr}
1 & H_{1} & H_{2} & H_{3} & \ldots \\
0 & -1 & -H_{1} & -H_{2} & \ldots \\
0 & 0 & 1 & H_{1} & \ldots \\
0 & 0 & 0 & -1 & \ldots \\
\vdots & \ddots & \ddots & \ddots & \ddots
\end{array}\right)
$$


Using Cramer's rule and then multiplying rows $2,3, \ldots n$ of the resulting determinant by $n, n-1, \ldots, 1$ respectively, we obtain

$$
\tilde{e}_{n}=\frac{1}{n !}\left|\begin{array}{cccccc}
\tilde{h}_{0} & \tilde{h}_{1} & \tilde{h}_{2} & \cdots & \tilde{h}_{n-1} & \tilde{h}_{n} \\
n & n H_{1} & n H_{2} & \cdots & n H_{n-1} & n H_{n} \\
0 & n-1 & (n-1) H_{1} & \cdots & (n-1) H_{n-2} & (n-1) H_{n-1} \\
0 & 0 & n-2 & \ddots & (n-2) H_{n-3} & (n-2) H_{n-2} \\
\vdots & \vdots & \ddots & \ddots & \ddots & \vdots \\
0 & 0 & 0 & \cdots & 1 & H_{1}
\end{array}\right|
$$

We will finally show that by manipulating determinant (3.28), we obtain determinant (3.35). Let $R_{i}$ be row $i$ of determinant (3.28). If $R_{2} \rightarrow R_{2}+R_{3} h_{1}+\cdots+R_{n} h_{n-2}$ in this determinant, the second row becomes that of determinant (3.35) due to the simple identity (for $i=1, \ldots, n$ )

$n H_{i}=(n+i) h_{i}+(n+i-2) h_{i-1} h_{1}+\cdots+(n+i-2 i+2) h_{1} h_{i-1}+(n+i-2 i) h_{i}$.

Doing similar operations on the lower rows, the two determinants are seen to coincide.

\subsection{Power sums}

We define the $n$-th bosonic and fermionic power sums as follows:

$$
p_{n}:=\sum_{i=1}^{\infty} x_{i}^{n}=m_{(n)} \quad \text { and } \quad \tilde{p}_{n}:=\sum_{i=1}^{\infty} \theta_{i} x_{i}^{n}=m_{(n ; 0)} .
$$

Note that this time we will set $p_{0}=0$. Obviously,

$$
n \tilde{p}_{n-1}(x, \theta) \sim n \tilde{p}_{n-1}(x, d x)=d p_{n}(x)
$$

for all $n \geq 1$.

Proceeding as in the complete symmetric functions case, we introduce products of power sums:

$$
p_{\Lambda}:=\prod_{i=1}^{\bar{\Lambda}} \tilde{p}_{\Lambda_{i}} \prod_{j=\underline{\underline{\Lambda}}+1}^{\ell(\Lambda)} p_{\Lambda_{j}}
$$

Also, we find that the generating function for superpower sums is

$$
P(t, \tau):=\sum_{n \geq 0} t^{n} p_{n}+\tau \sum_{n \geq 0}(n+1) t^{n} \tilde{p}_{n}=\sum_{i=1}^{\infty} \frac{t x_{i}+\tau \theta_{i}}{1-t x_{i}-\tau \theta_{i}} .
$$


One directly verifies

$$
H(t, \tau) P(t, \tau)=\left(t \partial_{t}+\tau \partial_{\tau}\right) H(t, \tau)
$$

and

$$
E(t, \tau) P(-t,-\tau)=-\left(t \partial_{t}+\tau \partial_{\tau}\right) E(t, \tau) .
$$

These expressions lead after some manipulations to the following recursion relations.

Lemma 26. Let $n \geq 1$. Then

$$
h_{n}=\sum_{r=1}^{n} p_{r} h_{n-r}, \quad n e_{n}=\sum_{r=1}^{n}(-1)^{r+1} p_{r} e_{n-r} .
$$

Let $n \geq 0$, and recall that $p_{0}=0$. Then

$$
\begin{gathered}
(n+1) \tilde{h}_{n}=\sum_{r=0}^{n}\left[p_{r} \tilde{h}_{n-r}+(r+1) \tilde{p}_{r} h_{n-r}\right], \\
(n+1) \tilde{e}_{n}=\sum_{r=0}^{n}(-1)^{r+1}\left[p_{r} \tilde{e}_{n-r}-(r+1) \tilde{p}_{r} e_{n-r}\right] .
\end{gathered}
$$

Theorem 27. Let $\hat{\omega}$ be the involution defined in (3.21). Then, for $n>0$,

$$
\hat{\omega}: p_{n} \longmapsto(-1)^{n-1} p_{n} \quad \text { and } \quad \tilde{p}_{n-1} \longmapsto(-1)^{n-1} \tilde{p}_{n-1}
$$

or, equivalently,

$$
\hat{\omega}\left(p_{\Lambda}\right)=\omega_{\Lambda} p_{\Lambda} \quad \text { with } \quad \omega_{\Lambda}:=(-1)^{|\Lambda|+\underline{\bar{\Lambda}}-\ell(\Lambda)} .
$$

Proof: We use Lemma 26 and proceed as in the proof of Theorem 23 . 
Proposition 28. For $n \geq 1$, we have

$$
p_{n}=\left|\begin{array}{ccccc}
e_{1} & 2 e_{2} & 3 e_{3} & \cdots & n e_{n} \\
1 & e_{1} & e_{2} & \cdots & e_{n-1} \\
0 & 1 & e_{1} & \cdots & e_{n-2} \\
\vdots & \ddots & \ddots & \ddots & \vdots \\
0 & 0 & 0 & & e_{1}
\end{array}\right|, \quad n ! e_{n}=\left|\begin{array}{ccccc}
p_{1} & p_{2} & \cdots & p_{n-1} & p_{n} \\
1 & p_{1} & \cdots & p_{n-2} & p_{n-1} \\
0 & 2 & \ddots & p_{n-3} & p_{n-2} \\
\vdots & \ddots & \ddots & \ddots & \vdots \\
0 & 0 & \cdots & n-1 & p_{1}
\end{array}\right|
$$

For $n \geq 0$, we have

$$
\tilde{p}_{n}=\left|\begin{array}{ccccc}
\tilde{e}_{0} & \tilde{e}_{1} & \tilde{e}_{2} & \cdots & \tilde{e}_{n} \\
1 & e_{1} & e_{2} & \cdots & e_{n} \\
0 & 1 & e_{1} & \cdots & e_{n-1} \\
\vdots & \ddots & \ddots & \ddots & \vdots \\
0 & 0 & 0 & & e_{1}
\end{array}\right|, \quad n ! \tilde{e}_{n}=\left|\begin{array}{ccccc}
\tilde{p}_{0} & \tilde{p}_{1} & \cdots & \tilde{p}_{n-1} & \tilde{p}_{n} \\
n & p_{1} & \cdots & p_{n-1} & p_{n} \\
0 & n-1 & \ddots & p_{n-2} & p_{n-1} \\
\vdots & \ddots & \ddots & \ddots & \vdots \\
0 & 0 & \cdots & 1 & p_{1}
\end{array}\right| .
$$

Similar formulas for the complete symmetric functions are obtained by using the involution $\hat{\omega}$.

Proof: The proof is similar to that of Proposition 25.

The explicit formulas presented in Proposition 28 establish the correspondence between the sets $\left\{p_{n}, \tilde{p}_{n-1}\right\}$ and $\left\{e_{n}, \tilde{e}_{n-1}\right\}$. This implies, in particular, that $e_{\Lambda}=$ $\sum_{\Omega} c_{\Lambda \Omega} p_{\Omega}$ for uniquely determined coefficients $c_{\Lambda \Omega} \in \mathbb{Q}$. Note that $c_{\Lambda \Omega}$ is not necessarily an integer since, for instance, $e_{2}=p_{1}^{2} / 2-p_{2}$. Theorem 21 and Proposition 28 thus imply the following result.

Corollary 29. The set $\left\{p_{\Lambda}: \Lambda \vdash(n \mid m)\right\}$ is a basis of $\mathscr{P}_{(n \mid m)}^{S_{N}}(\mathbb{Q})$ if either $n \leq N$ and $m=0$ or $n<N$ and $m>0$. In particular, the set $\left\{p_{\Lambda}: \Lambda \vdash(n \mid m)\right\}$ is a basis of $\mathscr{P}_{(n \mid m)}^{S_{\infty}}(\mathbb{Q})$.

The power sums will play a fundamental role in the remainder of the article. For this reason, we will consider, from now on, only symmetric polynomials in superspace defined over the rational numbers (or any greater field):

$$
\mathscr{P}^{S_{\infty}}:=\mathscr{P}^{S_{\infty}}(\mathbb{Q}) \text {. }
$$

Remark 30. The elementary and power-sum superpolynomials appear to have been introduced first in [2]. The power-sum superpolynomials were rediscovered in [8] (Section 2.5) and the expressions for the three multiplicative bases were first given in [9]. 


\subsection{Orthogonality}

Let $n_{\lambda}(i)$ denote the number of parts equal to $i$ in the partition $\lambda$. We introduce a bilinear form, $\left\langle\langle\mid\rangle: \mathscr{P}^{S_{\infty}} \times \mathscr{P}^{S_{\infty}} \rightarrow \mathbb{Q}\right.$, defined by

$$
\left\langle\overleftrightarrow{p_{\Lambda}} \mid \overrightarrow{p_{\Omega}}\right\rangle:=z_{\Lambda} \delta_{\Lambda, \Omega}, \quad \text { for } \quad z_{\Lambda}:=z_{\Lambda^{s}}=\prod_{k \geq 1}\left[k^{n_{\Lambda^{s}}(k)} n_{\Lambda^{s}}(k) !\right]
$$

Proposition 31. Let $f$ and $g$ be superpolynomials in $\mathscr{P}_{\infty}$. Then $\langle\langle\overleftarrow{f} \mid \vec{g}\rangle$ is a scalar product, that is, in addition to bilinearity, we have

$$
\begin{array}{ll}
\langle\overleftarrow{f} \mid \vec{g}\rangle=\langle\langle\overleftarrow{g} \mid \vec{f}\rangle\rangle=\langle\langle\vec{g} \mid \overleftarrow{f}\rangle \quad & \text { (symmetry) } \\
\langle\langle\overleftarrow{f} \mid \vec{f}\rangle\rangle=0 \quad \forall \quad f \neq 0 & \text { (positivity). }
\end{array}
$$

Proof: The symmetry property is a consequence of Lemma 10. The positivity of the scalar product is proved as follows. By definition $z_{\Lambda}>0$ and by virtue of Corollary 29 , there is a unique decomposition $f=\sum_{\Lambda} f_{\Lambda} p_{\Lambda}$. Therefore $\langle\langle\overleftarrow{f} \mid \vec{f}\rangle\rangle=$ $\sum_{\Lambda} f_{\Lambda}^{2} z_{\Lambda}>0$.

Proposition 32. The involution $\hat{\omega}$ is an isometry.

Proof: Given that $\left\{p_{\Lambda}\right\}_{\Lambda}$ is a basis of $\mathscr{P} S_{\infty}$, for any symmetric polynomials $f$ and $g$, we have $f=\sum_{\Lambda} f_{\Lambda} p_{\Lambda}$ and $g=\sum_{\Lambda} g_{\Lambda} p_{\Lambda}$. Thus

$$
\begin{aligned}
\langle\overleftarrow{\hat{\omega} f} \mid \overrightarrow{\hat{\omega} g}\rangle & =\sum_{\Lambda, \Omega} f_{\Lambda} g_{\Omega}\left\langle\left\langle\overleftarrow{\hat{\omega} p_{\Lambda}} \mid \overrightarrow{\hat{\omega} p_{\Omega}}\right\rangle\right. \\
& \left.=\sum_{\Lambda} f_{\Lambda} g_{\Omega}(-1)^{|\Lambda|+\underline{\underline{\Lambda}}-\ell(\Lambda)}(-1)^{|\Omega|+\underline{\bar{\Omega}}-\ell(\Omega)}\left\langle\overleftarrow{p_{\Lambda}} \mid \overrightarrow{p_{\Omega}}\right\rangle\right\rangle \\
& =\sum_{\Lambda} z_{\Lambda} f_{\Lambda} g_{\Lambda}=\langle\langle\overleftarrow{f} \mid \vec{g}\rangle\rangle
\end{aligned}
$$

as claimed.

The following theorem is of particular importance since it gives Cauchy-type formulas for the superpower sums.

Theorem 33. Let $K=K(x, \theta ; y, \phi)$ be the bi-symmetric formal function given by

$$
K:=\prod_{i, j} \frac{1}{1-x_{i} y_{j}-\theta_{i} \phi_{j}} .
$$


Then

$$
K=\sum_{\Lambda \in \mathrm{SPar}} z_{\Lambda}^{-1} \overleftrightarrow{p_{\Lambda}(x, \theta)} \overrightarrow{p_{\Lambda}(y, \phi)}
$$

Proof: We have:

$$
\begin{aligned}
& \prod_{i, j} \frac{1}{1-x_{i} y_{j}-\theta_{i} \phi_{j}} \\
& \quad=\exp \left\{\sum_{i, j} \ln \left[\left(1-x_{i} y_{j}-\theta_{i} \phi_{j}\right)^{-1}\right]\right\} \\
& \quad=\exp \left\{\sum_{i, j} \sum_{n \geq 1}\left[\frac{1}{n}\left(x_{i} y_{j}+\theta_{i} \phi_{j}\right)^{n}\right]\right\} \\
& \quad=\exp \left\{\sum_{n \geq 1}\left[\frac{1}{n} p_{n}(x) p_{n}(y)\right]+\sum_{n \geq 0}\left[\tilde{p}_{n}(x, \theta) \tilde{p}_{n}(y, \phi)\right]\right\} \\
& \quad=\prod_{n \geq 1} \sum_{k_{n} \geq 0} \frac{1}{n^{k_{n}} k_{n} !}\left[p_{n}(x) p_{n}(y)\right]^{k_{n}} \exp \left[\sum_{n \geq 0} \tilde{p}_{n}(x, \theta) \tilde{p}_{n}(y, \phi)\right] .
\end{aligned}
$$

Considering Proposition 11, we find

$$
\prod_{i, j} \frac{1}{1-x_{i} y_{j}-\theta_{i} \phi_{j}}=\sum_{n, m \geq 0} \sum_{\substack{\lambda \in \operatorname{Part}_{s}(n) \\ \mu \in \operatorname{Part}_{a}(m)}}\left[z_{\lambda}^{-1} p_{\lambda}(x) p_{\lambda}(y) \overleftrightarrow{\tilde{p}_{\mu}(x, \theta)} \overrightarrow{\tilde{p}_{\mu}(y, \phi)}\right]
$$

This equation (together with Lemma 10) proves the theorem.

Remark 34. The inverse of the kernel satisfies:

$$
K(-x,-\theta ; y, \phi)^{-1}=\prod_{i, j}\left(1+x_{i} y_{j}+\theta_{i} \phi_{j}\right)=\sum_{\Lambda \in \mathrm{SPar}} \omega_{\Lambda} z_{\Lambda}^{-1} \overleftrightarrow{p_{\Lambda}(x, \theta)} \overline{p_{\Lambda}(y, \phi)}
$$

The proof of this result is similar to that of Theorem 33, apart from the presence of the coefficient $\omega_{\Lambda}=(-1)^{|\Lambda|+\underline{\bar{\Lambda}}-\ell(\Lambda)}$, which comes from the expansion of $\ln \left(1+x_{i} y_{j}+\right.$ $\left.\theta_{i} \phi_{j}\right)$. This shows that

$$
K(x, \theta ; y, \phi)=\omega^{(x, \theta)} K(-x,-\theta ; y, \phi)^{-1}=\omega^{(y, \phi)} K(-x,-\theta ; y, \phi)^{-1}
$$

where $\omega^{(x, \theta)}$ indicates that $\omega$ acts on the $(x, \theta)$ variables and similarly for $\omega^{(y, \phi)}$.

We now give two direct consequences of Theorem 33 . 
Corollary 35. $K$ is a reproducing kernel in the space of symmetric superfunctions:

$$
\left\langle\langle K(x, \theta ; y, \phi) \mid f(x, \theta\rangle\rangle=f(y, \phi), \quad \text { for all } \quad f \in \mathscr{P}^{S_{\infty}} .\right.
$$

Proof: If $f \in \mathscr{P}^{S_{\infty}}$, there exist unique coefficients $f_{\Lambda}$ such that $f=\sum_{\Lambda} f_{\Lambda} p_{\Lambda}$. Hence,

$$
\begin{aligned}
\langle\langle K(x, \theta ; y, \phi) \mid f(x, \theta)\rangle\rangle & =\sum_{\Omega, \Lambda} z_{\Omega}^{-1} f_{\Lambda}\left\langle\left\langle\overleftrightarrow{p_{\Omega}(x, \theta)} \mid \overrightarrow{p_{\Lambda}(x, \theta)}\right\rangle\right\rangle \overrightarrow{p_{\Omega}(y, \phi)} \\
& =\sum_{\Lambda} f_{\Lambda} \overrightarrow{p_{\Lambda}(y, \phi)}=f(y, \phi)
\end{aligned}
$$

as desired.

Corollary 36. We have

$$
\begin{aligned}
h_{n} & =\sum_{\Lambda \in \operatorname{SPar}(n \mid 0)} z_{\Lambda}^{-1} p_{\Lambda} \quad \text { and } \quad e_{n}=\sum_{\Lambda \in \operatorname{SPar}(n \mid 0)} z_{\Lambda}^{-1} \omega_{\Lambda} p_{\Lambda}, \\
\tilde{h}_{n} & =\sum_{\Lambda \in \operatorname{SPar}(n \mid 1)} z_{\Lambda}^{-1} p_{\Lambda} \quad \text { and } \quad \tilde{e}_{n}=\sum_{\Lambda \in \operatorname{SPar}(n \mid 1)} z_{\Lambda}^{-1} \omega_{\Lambda} p_{\Lambda} .
\end{aligned}
$$

Proof: Using the definition of the generating function $E(t, \tau)$, we first make the following correspondence:

$$
E(t, 0)=\sum_{n \geq 0} t^{n} e_{n}(x)=\left.K(-x, 0 ; y, 0)^{-1}\right|_{y=(t, 0,0, \ldots)} .
$$

Thus, from Theorem 33 and $\left.p_{\lambda}(y)\right|_{y=(t, 0,0, \ldots)}=t^{|\lambda|}$, we have

$$
\sum_{n \geq 0} t^{n} e_{n}(x)=\sum_{\lambda \in \operatorname{Par}} t^{|\lambda|} \omega_{\lambda} z_{\lambda}^{-1} p_{\lambda}(x) \Longrightarrow e_{n}=\sum_{\lambda \vdash n} z_{\lambda}^{-1} \omega_{\lambda} p_{\lambda}
$$

Then, we observe that

$$
\partial_{\tau} E(t, \tau)=\sum_{n=0}^{N-1} t^{n} \tilde{e}_{n}(x, \tau)=\left.\partial_{\tau} K(-x,-\theta ; y, \phi)^{-1}\right|_{\substack{y=(t, 0,0, \ldots) \\ \phi=(-\tau, 0, \ldots)}} .
$$

Theorem 33 and

$$
\left.p_{\Lambda}(y, \phi)\right|_{\substack{y=(t, 0,0, \ldots) \\
\phi=(-\tau, 0, \ldots)}}=\left\{\begin{aligned}
t^{|\Lambda|} & \text { if } \underline{\bar{\Lambda}}=0 \\
-\tau t^{|\Lambda|} & \text { if } \underline{\bar{\Lambda}}=1 \\
0 & \text { otherwise }
\end{aligned}\right.
$$


finally lead to

$$
\sum_{n \geq 0} t^{n} \tilde{e}_{n}(x, \tau)=\sum_{\Lambda, \underline{\bar{\Lambda}}=1} t^{|\Lambda|} z_{\Lambda}^{-1} \omega_{\Lambda} p_{\Lambda}(x, \theta) \Longrightarrow \tilde{e}_{n}=\sum_{\Lambda \in \operatorname{SPar}(n \mid 1)} z_{\Lambda}^{-1} \omega_{\Lambda} p_{\Lambda} .
$$

Note that the minus sign disappears since $\partial_{\tau}$ and $p_{\Lambda}(x, \theta)$ anticommute when $\bar{\Lambda}=$ 1. Similar formulas relating the superpower sums to the homogeneous symmetric polynomials are obtained using the involution $\hat{\omega}$.

Lemma 37. Let $\left\{u_{\Lambda}\right\}$ and $\left\{v_{\Lambda}\right\}$ be two bases of $\mathscr{P}_{(n \mid m)}^{S_{\infty}}$. Then

$$
K(x, \theta ; y, \phi)=\sum_{\Lambda} \overleftrightarrow{u_{\Lambda}(x, \theta)} \overrightarrow{v_{\Lambda}(y, \phi)} \Longleftrightarrow\left\langle\overleftrightarrow{u_{\Lambda}} \mid \overrightarrow{v_{\Lambda}}\right\rangle=\delta_{\Lambda, \Omega} .
$$

Proof: The proof is identical to the one in the case without Grassmannian variables (see [13], I.4.6).

Proposition 38. Let $K$ be the function defined in (3.53). Then,

$$
K=\sum_{\Lambda \in \mathrm{SPar}} \overleftrightarrow{m_{\Lambda}(x, \theta)} \overrightarrow{h_{\Lambda}(y, \phi)}
$$

Proof: We start with the definition of the generating function $E(t, \tau)$ :

$$
\begin{aligned}
K(-x,-\theta ; y, \phi)^{-1} & =\prod_{i, j}\left(1+x_{i} y_{j}+\theta_{i} \phi_{j}\right)=\prod_{i} E\left(x_{i}, \theta_{i}\right) \\
& =\prod_{i}\left[\sum_{n \geq 0} x_{i}^{n} e_{n}(y)+\theta_{i} \sum_{n \geq 0} x_{i}^{n} \tilde{e}_{n}(y, \phi)\right] \\
& =\sum_{\epsilon_{1}, \epsilon_{2}, \cdots \in\{0,1\}} \sum_{n_{1}, n_{2}, \cdots \geq 0} \prod_{i}\left(\theta_{i}^{\epsilon_{i}} x_{i}^{n_{i}} e_{n_{i}}^{\left(\epsilon_{i}\right)}(y, \phi)\right) \\
& =\sum_{\Lambda \in \operatorname{SPar}} \overleftrightarrow{m_{\Lambda}(x, \theta)} \frac{e_{\Lambda}(y, \phi)}{\longrightarrow} .
\end{aligned}
$$

In the third line we have set $e_{n}^{(0)}(y, \phi)=e_{n}(y, \phi)$ and $e_{n}^{(1)}(y, \phi)=\tilde{e}_{n}(y, \phi)$. The fourth line follows by reordering the variables using Lemma 10. Using (3.58), we can recover $K(x, \theta ; y, \phi)$ by acting with $\hat{\omega}^{\{y, \phi\}}$ on $K(-x,-\theta ; y, \phi)^{-1}$. The identity then follows from $\hat{\omega}\left(e_{\Lambda}\right)=h_{\Lambda}$.

The previous proposition and Lemma 37 have the following corollary.

Corollary 39. The monomials are dual to the complete symmetric functions in superspace:

$$
\left\langle\overleftrightarrow{h_{\Lambda}} \mid \overrightarrow{m_{\Omega}}\right\rangle=\delta_{\Lambda, \Omega}
$$




\section{Concluding remarks}

Most of the key classical concepts in the theory of symmetric functions have been extended to superspace. The notable exception concerns the Schur function $s_{\lambda}$. The proper superspace generalization of the classical definition of Schur functions as a bialternant has not been found yet. (We point out in that regard that division by anticommuting variables is prohibited). Similarly, the Jacobi-Trudi identity, which expresses the Schur functions in terms of the $h_{n}$ 's, has not been generalized. Notice that in all the instances where we have obtained a determinantal expression, we had at most one row or one column made out of fermionic quantities, something which cannot be the case for the sought for Jacobi-Trudi super-identity. To determine whether these properties are specific to the $m=0$ sector or not requires further study.

Note also that, off-hand, it appears unlikely that the yet-to-be-defined Schur functions in superspace would be related to the representation theory of special Lie superalgebras since these theories do not involve Grassmannian variables. (Recall in that regard that the special importance of the Schur functions lies in their deep representation theoretic interpretation: $s_{\lambda}$ is a Lie-algebra character, being expressible as a sum of semistandard tableaux of shape $\lambda$.) Actually, it could well be that for the Schur superpolynomials, the representation theoretic interpretation is simply lost.

The simplest way of defining the Schur superpolynomials is by a specialization of the Jack superpolynomials. The latter have been defined in [6] as the unique polynomials in $\mathscr{P}^{S_{N}}$ which are: (1) eigenfunctions of supersymmetric differential operators; (2) triangular in the monomial basis with respect to the Bruhat order. It turns out that the Jack polynomials in superspace are also orthogonal with respect to a one-parameter deformation of the scalar product introduced in Section 3.4. This will be considered in a forthcoming article [10].

In a different vein, with the introduction of diagrams with circles, we expect a large number of results linked to "Ferrers diagram combinatorics" to have nontrivial extensions to the supercase. Pieces of "supercombinatorics" have already been presented at the end of Section 2.4.

Acknowledgements We thank A. Joyal for pointing out the connection between $\tilde{e}_{n-1}, \tilde{h}_{n-1}$ and $n \tilde{p}_{n-1}$ and the corresponding one-forms $d e_{n}, d h_{n}$ and $d p_{n}$. This work was supported by NSERC and FONDECYT (Fondo Nacional de Desarrollo Científico y Tecnológico) grant \#1030114. P.D. is grateful to the Fondation J.A.-Vincent for a student fellowship and to NSERC for a postdoctoral fellowship.

\section{References}

1. F. Brenti, "Francesco determinants of super-Schur functions, lattice paths, and dotted plane partitions," Adv. Math. 98 (1993), 27-64.

2. L. Brink, A. Turbiner, and N. Wyllard, "Hidden algebras of the (super) Calogero and Sutherland models," J. Math. Phys. 39 (1998), 1285-1315.

3. S. Corteel and J. Lovejoy, “Overpartitions," Trans. Amer. Math. Soc. 356 (2004), 1623-1635.

4. P. Desrosiers, L. Lapointe, and P. Mathieu, "Supersymmetric Calogero-Moser-Sutherland models and Jack superpolynomials," Nucl. Phys. B606 (2001), 547-582.

5. P. Desrosiers, L. Lapointe, and P. Mathieu, "Jack superpolynomials, superpartition ordering and determinantal formulas," Commun. Math. Phys. 233 (2003), 383-402. 
6. P. Desrosiers, L. Lapointe, and P. Mathieu, "Jack polynomials in superspace," Commun. Math. Phys. 242 (2003), 331-360.

7. P. Desrosiers, L. Lapointe, and P. Mathieu, "Explicit formulas for the generalized Hermite polynomials in superspace," J. Phys. A37 (2004), 1251-1268.

8. P. Desrosiers, L. Lapointe, and P. Mathieu, "Generalized Hermite polynomilas in superspace as eigenfunctions of the supersymmetric rational CMS model," Nucl. Phys. B674 (2003), 615-633.

9. P. Desrosiers, L. Lapointe, and P. Mathieu, "Supersymmetric Calogero-Moser-Sutherland models: Superintegrability structure and eigenfunctions," in Superintegrability in Classical and Quantum Systems, P. Tempesta et al. (Eds.), CRM Proceedings and Lecture Notes, Vol. 137 (2004), pp. 109-124.

10. P. Desrosiers, L. Lapointe, and P. Mathieu, "Jack polynomials in superspace: combinatorial orthogonality," submitted, math-ph/050939.

11. P.H. Dondi and P.D. Jarvis, "Diagram and superfield techniques in the classical superalgebras," J. Phys. A14 (1981), 547-563.

12. V.G. Kac, "Characters of typical representations of classical Lie superalgebras," Comm. Algebra 5 (1977), 889-897.

13. I.G. Macdonald, Symmetric functions and Hall polynomials, 2nd edition, The Clarendon Press/Oxford University Press (1995).

14. L. Manivel, "Symmetric functions, Schubert polynomials, and degeneracy loci," American Mathematical Society (2001).

15. E.M. Moens and J. van der Jeugt, "Determinantal formula for supersymmetric Schur polynomials," $J$. Algebraic Combin. 17 (2003), 283-307.

16. I. Pak, Partition Bijections, a Survey, to appear in Ramanujan Journal.

17. R.P. Stanley, "Enumerative combinatorics," Vol. 2, Cambridge Studies in Advanced Mathematics Vol. 62, Cambridge University Press (1999).

18. J.R. Stembridge, "A characterization of supersymmetric polynomials," J. Alg. 95 (1985), 439-444. 